\title{
PONDERACIÓN Y JUSTO MEDIO
}

\section{Autor: Elina lbarra}

Graduada en la Facultad de Filosofía y Le-tras de la Universidad de Buenos Aires, Argentina. Actualmente es Investigadora Adscrita en el Instituto de Investigaciones Jurídicas "A m-brosio Gioja". Profesora adjunta de Teoría del Estado y de Teoría General y Filosofía del Derecho en la Facultad de Derecho de la Universidad de Buenos Aires, en Argentina. Profesora de Fundamentos de Bioética en la Carrera de Actualización en Bioé-tica y en la Maestría en la misma Facultad. Profesora Adjunta de Ética, Directora de Proyectos de Investigación y Coordinadora de la Licenciatura en Ciencia Política de la Universidad Abierta Interamericana. Correo electrónico: chinacruel@hotmail.com.

https://orcid.org/0000-0002-3852-0832

\section{(Deliberation and just measure)}

Fecha de recepcion: 28 de enero de 2017 Fecha de aceptacion: 30 de marzo de 2017

Resumen: El presente escrito tiene como objetivo establecer puntos de contacto entre la teoría del Justo Medio aristotélico y la Ponderación propuesta por Robert Alexy. Entre ambas propuestas existen tanto semejanzas como diferencias, entre lo que considero dos metodologías algorítmicas en la determinación de un equilibrio, considerado como criterio para el establecimiento de "lo justo". Tanto la teoría del Justo medio aristotélica, que permite arribar, a través de un equilibrio, resultado de la medida justa entre dos extremos igualmente viciosos, a las acciones prescriptas que permitirán llegar a ser y a ser visto por los pares, como un ciudadano virtuoso; así también la Ponderación, considerada una metodología que, en el contexto de una confrontación de principios, permite establecer cuál es la medida inmejorable en la que cada uno debe ser considerado. Si bien, las líneas argumentales son disímiles, puesto que son dos concepciones que se hayan a veinticinco siglos de distancia temporal, cultural, hay una noción de justicia que permanece, en la forma de que es posible hallar $y$, en un punto tal que si se modifica en mayor o en menor medida, se corre el riesgo de perder de vista el punto óptimo, que representa el punto justo.

Palabras clave: Aristóteles, Justo Medio, Alexy, Ponderación, Justicia, Principios, Virtud

\begin{abstract}
The aim of this paper is to establish points of contact between the theory of "just measure" of Aristotle and the "weighting" of Robert Alexy. Among these proposals there are both similarities and differences: I consider that both proposals are algorithmic methodologies in determining a balance, and are tools used as a criterion for establishing "the justice". Thus, both the theory of Aristotelian "just measure", which allows us to reach the right amount of the prescribed actions that allow us to become a virtuous citizen, as the weighting, a methodology that considered in the context of a confrontation of principles, allows for what is the unbeatable extent to which each should be considered. While the storylines are dissimilar, since they are two concepts that have been twenty-five centuries of temporal, cultural distance is a notion of justice that remains in the way that it is possible to find and point that if modified in greater or lesser extent lost sight of the point of optimization, which represents the right point.
\end{abstract}

Keys word: Aristotle, Just Measure, Alexy, weighting, Justice Principles, Virtue 


\section{Parte I}

$E$ I problema de la Justicia ha atravesado todas las esferas de la historia del pensamiento humano. Está presente desde el comienzo mismo de la racionalidad occidental, que se dio a partir de una traducción logo-sófica de un mundo que anteriormente era gobernado por el capricho de deidades, según la visión del mundo desde el imperio del mito. Por lo tanto, la justicia de entonces estaba teñida no de un criterio único, sino que estaba sometida a los pareceres antojadizos de las voluntades siempre volubles de los dioses. Así la Justicia, tenía aspecto de tragedia: un destino ineludible que rodeaba los acontecimientos de un áura de sinsentido - lo dionisíaconietzcheano - lo indecible de sus razones, no sólo era un llamado de lo irracional a lo indecible, sino también a lo indecifrable e impredecible.

La primera producción sistemática en torno a la Justicia, se dará en el pensamiento platónico, expresado en su obra más completa en torno a este tema y también, quizá, la más compleja, $R e$ pública ${ }^{1}$.Aunque es posible afirmar que la preocupación de Platón por el problema de la Justicia, pudo ser su motivación más profunda, resultado de la muerte de Sócrates. Por ello es posible afirmar que estuvo tratando este problema a lo largo de toda su obra. En República se discurre en torno a la búsqueda del orden político ideal, y efectivamente Platón da cuenta de él, poniendo en boca de Sócrates su diseño y su fundamento. Ante tal descripción Adimanto - uno de sus interlocutores - le reprocha que tal orden, si bien habrá de ser justo, los hombres en él no serían felices porque consistía en un orden profundamente sacrificado y poco grato al placer. Sócrates responde que, el objetivo era pensar en un orden justo para todos y no en un orden feliz, y que si fuera posible una mó $\lambda$ ıs justa y feliz - y esa será la idea a explorar en los seis libros restantes- habría que ver si esa felicidad sería para todos la misma. Claramente lo que este planteo deja expuesto es la inadecuación entre orden justo y felicidad, o al menos, indica que no han de co-implicarse necesariamente. Lo central de este escrito es que la Justicia ya no será el resultado del dócil sometimiento a la voluntad caprichosa de alguna deidad, como así tampoco de ningún hombre. Lo justo puede ser alcanzado por los hombres que se ejercitan en la razón, y se subordinan a su sabiduría.

En el pensamiento helénico las esferas de la existencia no estaban escindidas, sino que lo bueno, lo verdadero, lo bello y lo justo eran considerados todos atributos co-implicados, tal que lo bueno habría de ser también bello y también justo. Pero la idea de que la justicia no ha de acarrear necesariamente la felicidad atraviesa todo el mundo antiguo, desde la Ley del Talión, pasando por Antígona -en ella está representada toda la dinámica de la tragedia griega - la cual aparece como un sucedáneo bastante difuso de la justicia divina del dios cristiano que impera en los once siglos de la Edad Media. Claramente tal asociación no se daba de manera lineal, sino que requería de un aparato argumental profundo y del que la ontología debía dar cuenta.

Así, si bien tanto para Platón como para Aristóteles, la Justicia es una de las virtudes cardinales - sabiduría, valentía y templanza - en tanto es ella la que gobierna a todas las otras virtudes y hace posible que se complementen armoniosamente en cada uno de los hombres; es también la virtud más específica, ya que ella es condición de que se realice lo más beneficioso para todos. ${ }^{2} Y$ es por ello que no puede ser considerada sólo una virtud, sino que también se la reviste de cierta necesidad fundamental para la institución política, es decir, para la vida en la polis. Esta prioridad de la Justicia debe ser considerada en un sentido limitado, en tanto la centralidad del carácter social del hombre, y en torno a una noción del bien común pensado desde una visión comunitarista del orden político.

1- Platón, República, Buenos Aires, Eudeba, 1973, , Libro IV, 419a - 421c.

2- Aristóteles, Ética Nicomaquea, Madrid, Gredos, 2007, Libro V, 1129a - 1138b. 


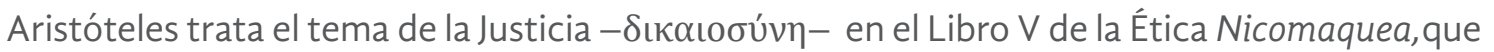
se llama Sobre las Virtudes Éticas, donde la Justicia es considerada una especie de disposición que permite a los hombres ser más aptos para cumplir con las acciones justas, los lleva a obrar justamente y a desear las cosas justas. Lo inquietante es que la misma palabra se utiliza con diferentes acepciones cada vez. Pero la Justicia también es por sobre todas las cosas el Justo Medio - $\mu \varepsilon \sigma o \tau \eta s$, en Griego- ya sea en cualquiera de las modalidades antes indicadas. Porque el Justo Medio es el criterio metodológico que va a permitir establecer la medida justa en que tales repartos y atribuciones han de ser distribuidos y/o asignados. Se trate de:

- justicia particular: cuando su función es la de regir los repartos o los intercambios de bienes.

- justicia universal: se refiere a la observancia de las leyes.

- justicia distributiva: cuando se ocupa de repartir los bienes entre las personas en relación con los méritos - la vida política antigua tenía un fuerte componente meritocrático.

- justicia reparadora: rige las transacciones en las que las partes han de ser consideradas iguales, en busca de rectificar el perjuicio causado, y para ello se debe tener en cuenta el daño y la intención.

- justicia conmutativa: gobierna las relaciones comerciales y tiene como fundamento la institución de la moneda, porque esta permite un valor común para establecer la justeza de los intercambios.

En el contexto del pensamiento aristotélico, también es posible distinguir entre:

- justicia natural: que es la que mueve a todo lo que existe y que

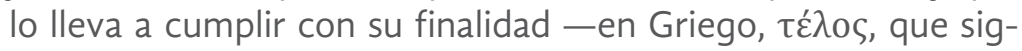
nifica: fin hacia el que se tiende-y que no depende de la voluntad humana, y que ha de ser siempre invariable. Es la que se expresa en la regularidad de la dinámica natural de los ciclos.

- justicia positiva: es la que rige en cada uno de los órdenes políticos. Como vemos esta distinción se corresponde con la innovación de los sofistas que diferenciaban entre $\varphi \ddot{\sigma} \iota \varsigma$ y vó $\mu$ os, como dos ámbitos con dinámicas diversas, e indicaban la impertinencia de adjudicar la propiedad de justo o injusto al ámbito natural, puesto que carece de voluntad o libertad para poder discernir diferentes cursos de acción, a diferencia del hombre.

La respuesta de Aristóteles es que las reglas del derecho no se fundan en la naturaleza sino en la voluntad de los hombres, y esto se ve claramente dada la diversidad de órdenes políticos que los hombres se han dado, pero que el orden justo presenta sólo una forma de gobierno y que será en todas partes el mejor o tendiente a él. ${ }^{3}$ Es que el pensamiento aristotélico tiene como supuesto que toda actividad humana tiende a un fin, y el fin siempre es superior, mejor y preferible al medio instrumentado para obtenerlo.Si hay algún fin querido totalmente en sí mismo, no como 
medio para otra cosa, este fin superior debe ser objeto de la disciplina Política: la Ética es parte de la Política; y no puede ser una ciencia exacta, porque no trata de las esencias - que son inmutables- sino de las acciones humanas. Además el fin de la Política -y por ende de la Ética- no es el conocimiento sino la acción; por lo que poco vale el conocimiento si se lo dirige a quien no está en condición de asimilarlo prácticamente. En cambio para quien sea maduro y racional, siempre será provechoso saber de estas cosas.

Para Aristóteles la meta de la Política es lograr el bien supremo, que es la felicidad —en Griego,

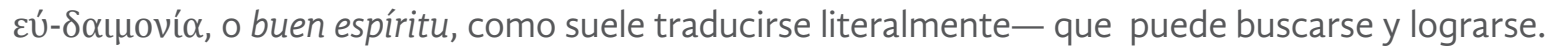
Todos están de acuerdo en que buscan la felicidad, pero no hay acuerdo acerca de en qué consiste. $\mathrm{Ni}$ el placer, ni los negocios ni los honores pueden ser la felicidad —aunque muchos lo crean así; el placer bestializa, es pasivo; los negocios buscan dinero, que es un medio; los honores parecen valorar más a quien los prodiga que a quien los recibe. Esto es la refutación de la idea platónica del bien: el bien se dice de muchas maneras y no puede ser lo mismo en todos los casos; además, el bien buscado no sólo es inalcanzable -en los términos platónicos- sino que además consiste en una postulación con serios problemas metodológicos.

La Política entonces, es el saber que busca procurar la felicidad para todos, y detrás de ello está la virtud cardinal de la justicia en su mayor dimensión. Pero la Política supone otro saber, sobre el que se funda, y que es la Ética, como el saber que se ocupa de procurar la felicidad para cada uno de los hombres. Como vemos son saberes dependientes uno del otro. Esto no debe perderse de vista cuando en la Ética Nicomaquea, Aristóteles señala que el ejercicio de la virtud es la manera de lograr la felicidad, porque esta sólo puede encontrarse en la virtud, ya que

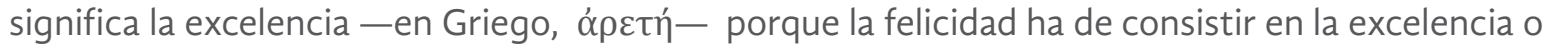
perfección de la función que le es propia al hombre: su racionalidad. ${ }^{4}$

Como el modo de ser es resultado de la realización de ciertas acciones, debemos investigar qué tipo de acciones llevan al modo de ser bueno. Esto siempre será esquemático, pues no se trata de saber qué es bueno, sino de ser buenos. La virtud no es una pasión ni una facultad, sino un "modo de ser": La virtud es el modo de ser por el cual nos comportamos bien o mal respecto de nuestras pasiones: si nos comportamos débilmente o desmesuradamente con nuestras pasiones, obramos mal; si nos comportamos moderadamente, obramos bien con ellas. Si esto es correcto, la virtud de hombre consiste en el modo de ser por el cual el hombre realiza su propia función como hombre; y eso no es otra cosa que buscar el justo medio o proporción en todo, lo que significa, estar determinado por la razón.Pero se trata del justo medio "en relación a cada uno" y "en relación con cada situación".

En la naturaleza de las cosas está el ser destruidas por defecto o por exceso, y se conservan por la proporción y el término medio. Lo mismo ocurre con las virtudes. Hacer algo justo no me hace justo, pues se requiere además: saber que se hace justicia; elegir hacerla; y hacerla con firmeza e inquebrantablemente.Siempre hay tres "disposiciones", dos de las cuales son "vicios" y una "virtud". El medio se opone a ambos extremos que son igualmente viciosos, pero no siempre del mismo modo: el uno es vicioso por defecto, es decir, por carencia de virtud, y el otro es vicioso por exceso, es decir por un desborde de la capacidad. Esto ocurre por la cosa misma, o porque nosotros somos más proclives a lo que nos da placer que al dolor, y nos inclinamos más fácilmente a los vicios del placer que a los otros.

Entonces las condiciones que hacen de nuestras acciones, actos virtuosos, es decir, actos justos —en su justa medida, esto es, relativos al que realiza la acción y a la situación- son:

- acciones que resultan de una toma de decisión, de una elección racional, es decir que fueron mediadas por una deliberación, por

4- Idem/. 1096a-1907b. 
la consideración teorética de los elementos que entran en juego en ella;

- acciones que son habituales, es decir, que no se dan ni casual, ni esporádicamente, sino que son frecuentes y regulares en su realización. Es Aristóteles el autor de la expresión "Una golondrina no hace verano" ${ }^{5}$, porque una acción por sí sola no hace a alguien virtuoso, sino el ejercicio cotidiano de ella, ha de ser el resultado de una práctica permanente, una manera de obrar constante, de lo que se ha vuelto una costumbre en nosotros y así revela nuestro carácter.

- las acciones han de hallarse en una posición intermedia entre el exceso y el defecto de una virtud, es decir que debe ser una acción que implique no caer en ninguno de los dos extremos, porque son igualmente viciosos.

- esas acciones estarán determinadas por la prudencia, ya que, la acción que resulta el justo medio entre dos extremos, no será una regla o una norma, sino que resultará de una visión concreta de la situación. Depende el justo medio de una deliberación relativa a nosotros en cada caso y a las circunstancias.

La dimensión política está implícita en el justo medio, ya que siempre se requiere de otra persona respecto de la cual poder tener la ocasión de ser justos.

\section{Parte II}

Históricamente el problema de la justicia ha girado en torno a su administración, es decir, en relación con los criterios de distribución de derechos y obligaciones y de cómo establecer la corrección y pertinencia de tal adjudicación, y la justificación de esas decisiones. La justicia asociada a la existencia de una respuesta correcta fue planteada por Ronald Dworkin, en su obra Los derechos en serio, ${ }^{6}$ donde se distingue de las respuestas dadas por las Teorías Positivistas como las de Hart y Kelsen. Alexy, en el contexto de esta discusión en torno a la posibilidad de la existencia de una única respuesta correcta, sostiene que una teoría de los principios no puede sostener por sí sola esta tesis.

Lo cierto es que la tesis de Dworkin sólo puede sostenerse bajo dos supuestos: el de la equiparación entre normas y principios -que Alexy discutirá- y la postulación del llamado Juez Hércules, cuya habilidad, sabiduría y paciencia sin límites hará posible que soporte sobre sus espaldas la pirámide invertida del sistema normativo. Para Alexy tal posibilidad, tal y como lo plantea Dworkin es insostenible, porque si bien tanto las reglas como los principios son normas, se aplican de manera diferente, porque tienen diferentes características:

- en cuanto a la aplicabilidad, la regla se aplica de todo o nada; en cambio el principio no determina completamente la decisión, sólo da razones para su aplicación

- en cuanto a la validez, la regla es válida o no es válida y por lo

5-Idem/. 1098a.

6- Dworkin, Ronald Los derechos en serio, Barcelona, Editorial Ariel, 1984. 
tanto, será aplicable o no; en cambio los principios pueden colisionar y ser válidos los dos al mismo tiempo

- en cuanto a la lógica, las reglas respetan el Principio de No Contradicción, "No es posible que A y $\neg \mathrm{A}$ sean ambas verdaderas y pertenezcan al mismo sistema normativo"; en cambio los principios se rigen por el Principio del Tercero Excluido, "No es posible que $A \circ \neg A$ no sean proposiciones del sistema, al menos una de ellas ha de serlo, excluyendo cualquier posibilidad respecto de su verdad que no sea $A \circ \neg A^{\prime \prime}$

Los principios son normas que ordenan que se realice algo en toda su potencialidad, es decir, que se realice en grado máximo, en relación con las posibilidades jurídicas y fácticas. Por ello, son llamados "mandatos de optimización", los cuales deben ser cumplidos en grado sumo, si es que estos permiten discernir entre diversos grados de cumplimiento. La medida de su realización no sólo depende de los condicionamientos fácticos de su aplicación, sino también de las posibilidades jurídicas, esto es, en relación con las normas que juegan en "sentido contrario" o en tensión. Se define "tensión" como la condición de equilibrio en el que no hay supremacía clara de uno frente al otro de los extremos en pugna. ${ }^{7}$

Robert Alexy propone que esas circunstancias reclaman un tipo de procedimiento de resolución diferente al utilizado para dirimir entre reglas opuestas, dada la naturaleza diversa de los principios. Esta propuesta metodológica, encuentra en la ponderación el mecanismo que permitirá, no ya subsumir ni dirimir uno por otro, sino adjudicar proporcionalmente al caso en cuestión grados de cumplimiento de los principios contrapuestos.

En la ponderación el "factor decisivo lo constituye el principio al que le corresponde un peso relativamente mayor en el caso concreto. Al principio que juega en sentido contrario se le hace retroceder, pero no se le declara inválido." 8

Esta característica de los principios de consistir en una exigencia de una determinada medida de cumplimiento tal que sea esta óptima, los diferencia claramente de las normas. Pero también, implica que la tarea de aplicación, en cuanto a su forma jurídica, es siempre en el fondo, también una tarea moral, a causa no sólo del contenido de los principios, sino también en su aplicación, dado que en ella está contenido un imperativo que manda "procurar su cumplimiento en la mayor medida posible". Este mandato conlleva en sí una carga moral innegable, en su búsqueda de mejorar las condiciones de la existencia, en virtud de un horizonte en el que se cristaliza una idea regulativa de bien.

Pero la consecuencia más inquietante es que a través de los principios, fundamentalmente en los principios constitucionales, es donde podemos afirmar que la tesis de las Teorías Positivistas referidas a la división tajante entre Derecho y Moral quedan rebatidas. La relación entre Derecho y Moral deben ser pensadas desde la vinculación ineludible en la aplicación de principios entre, la argumentación jurídica y la moral.

En cuanto a los principios y la relación entre ellos, no es posible decir que tengan valores o pesos unos más que otros, pues intentar establecer una jerarquía sería muy difícil, ya que su peso es relativo y por lo tanto las relaciones de prioridad que podamos entablar han de ser siempre pensadas en relación con un caso puntual y sus circunstancias. Los principios en su con-

7- Alexy, Robert "Sistema Jurídico, principios jurídicos y razón práctica", en Doxa 5, Universidad de Alicante, 1988, traducción de Manuel Atienza.

8-Alexy, Robert Derecho y razón práctica, México, Editorial Fontamara, 1993, pág. 13. 
junto no serían más que un catálogo de puntos de vista o de tođóı, los cuáles pueden decirnos qué se debe tomar en consideración, más no pueden establecer con univocidad qué esperan como resultado de su aplicación. Este es su talón de Aquiles, es la variable más débil de esta teoría de los principios, su nivel de abstracción y generalidad que los vuelven maleables y versátiles, a la vez los revela difusos, amplios e imprecisos.

En este punto hay divergencia, ya que unos podrían considerarlo una ventaja para otros es una deficiencia. Pero, es importante señalar lo siguiente: aquello que permite resolver el conflicto es precisamente esa fuerza de fluctuación en el peso relativo entre los principios. Este hecho se confronta en una tensión de principios, y a esa diferencia es lo que se denomina "valor". Entonces en lugar de afirmar que se da una colisión de principios, debería decirse, en cambio que, hay una colisión entre valores. Una de las consecuencias que esto produce es una pluralidad de modos diferentes de resolverse la cuestión de maneras más adecuadas. Y esto es a la vez, otro argumento en auxilio de la pretensión de sostener que las relaciones de prioridad entre los principios se corresponde con el problema de la jerarquía de los valores. ${ }^{9}$

Y si bien no es posible establecer un orden estricto entre los principios de acuerdo a su jerarquía, si en cambio es posible establecer un orden débil teniendo en cuenta los siguientes tres elementos:

- un sistema de condiciones de prioridad: las colisiones entre principios han de resolverse mediante ponderación en los casos concretos, pero esto no significa que la solución sea significativa solamente para ese caso, sino que pueden establecerse como antecedentes para ser considerados en futuros casos concretos. Por esta razón es que también la ponderación de principios supone o lleva implicada consigo la posibilidad de un procedimiento de argumentación que, lleva implicada consigo la posibilidad de un procedimiento de argumentación sin tales colisiones, el que estaría enmarcado dentro de una teoría de la argumentación.

- un sistema de estructura de ponderación: los principios en cuanto mandatos de optimización exigen una realización lo más completa dentro de sus potencialidades, en relación con las posibilidades jurídicas y fácticas. Las posibilidades fácticas están relacionadas con la capacidad de adecuación y necesidad. En cambio, las posibilidades jurídicas implican una ley de ponderación que puede ser formulada así: cuanto más alto sea el grado de incumplimiento o de menoscabo de un principio, tanto mayor debe ser la importancia del cumplimiento del otro. De esta manera no hace más que enunciar un principio de proporcionalidad en sentido estricto. ${ }^{10}$

- un sistema de prioridades prima facie: son los elementos que establecen cargas en la argumentación y de esta manera crean cierto orden entre los principios, pero que no implican una determinación, ni obligan en esa jerarquía ocasional establecida; sólo rigen en el campo de los principios. Este orden estará supeditado

9-Idem/.Pág. 16.

10- Idem/. Pág. 18. 
a nuevas argumentaciones que irán reacomodándose de acuerdo

a las cargas de las pruebas de cada caso.

Es por ello que en la cuestión respecto de la única respuesta posible a la resolución de un caso jurídico, habría que responder con una reformulación del planteo, indicando que quizá sea más apropiado hablar de "la mejor respuesta posible" en relación con la colisión entre principios. Ya que esta no depende de que haya una metodología que permita acceder a ella, sino en realidad viene a completar el modelo del sistema jurídico, porque tanto los principios como las reglas no regulan por sí mismos su aplicación.

La centralidad de una teoría de la argumentación jurídica es irrebasable, ya que se trata en definitiva de poder dar una base racionalmente fundamentada a las decisiones en torno a la colisión entre principios. Sobre todo debe considerarse la implicancia práctica de estas resoluciones y de las premisas de la argumentación que operará como fundamento, es por ello que será imprescindible como parte de este procedimiento el señalamiento de las buenas razones que dan apoyo a la decisión. La racionalidad de la argumentación jurídica depende en gran medida de que esas valoraciones que entraron en juego en la argumentación sean susceptibles de un control racional. ${ }^{11}$

\section{Parte III}

Para quien haya seguido en lectura atenta la descripción de ambas metodologías orientadas a establecer el punto óptimo de una acción a ser llevada a cabo dentro de un horizonte de cristalizaciones de la idea de bien en el contexto de una comunidad, habrá visto los numerosos puntos de contacto entre ellas. Tanto Aristóteles como Alexy sitúan el problema moral y el político-jurídico - es decir, la administración de justicia- a muy corta distancia una de otra, es más, indican claramente una co-implicación entre ambos órdenes, cuando no, la arbitrariedad de la distinción tajante entre uno y otro.

Indicaré tres puntos de contacto claros entre ambas teorías, salvando las distancias y diferencias de enfoques y finalidades que pueda haber entre ambos pensadores:

- Virtudes y Principios: hemos señalado que Aristóteles define a la virtud como la excelencia o la expresión en grado superior de una capacidad y/o habilidad, cuya expresión o puesta en práctica requiere de que ella se manifieste siempre entre dos extremos igualmente viciosos, tanto que puede ser definida como "el hábito de elección, que consiste en una posición intermedia relativa nosotros, determinada por la razón y por tal como la determinaría el hombre prudente."12 Vemos que la definición al igual que la de los principios como "mandatos de optimización que exigen el grado más alto de cumplimiento", es semejante a la aristotélica. Para empezar, no son definiciones sustanciales, sino formales, ya que establecen las condiciones en las que se ha de lograr el acto virtuoso o la decisión justa. En las dos definiciones se establece el

\footnotetext{
11= Idem/. Pág. 20

12- Aristóteles, Idem/. 1106b-1107a
} 
grado de optimización que define los elementos, en el caso de la virtud, que es la "excelencia" en grado supremo, implica siempre esta cualidad el estar de acuerdo a las circunstancias que la reclaman, por lo tanto es siempre un grado supremo en relación con las condiciones puntuales de la acción en cuestión. En el caso de los principios, estos han de ser considerados en la aplicación en relación con el caso concreto. Vemos que tanto principios como virtudes no tienen un valor sustantivo ni absoluto, sino formal y relativo a las condiciones de aplicación. Una distinción a tener en cuenta es que entre las virtudes suele haber una jerarquización, por ejemplo entre virtudes éticas y dianoéticas, pero todas están supeditadas a la virtud cardinal que es la Justicia. Y me permito decir que todos los principios, si bien carecen de un orden jerárquico fuerte, están todos en función de una decisión "justa”.

- Justo medio y ponderación: para Aristóteles la virtud ética superior es la Justicia, no sólo eso, sino que podríamos decir que es la virtud misma, pero esta resulta de un equilibrio entre los dos extremos que expresan, por un lado la carencia de virtud y por el otro el exceso de esa cualidad. Es decir que el justo medio -la expresión de justicia aristotélica que funda la cohesión social, base de la convivencia en la polis- es el punto máximo en el que una virtud puede expresarse en esas circunstancias, y siempre en la tensión entre dos extremos opuestos, tanto que si me acerco a uno, me alejo del otro. En este punto la ponderación está muy cercana al justo medio aristotélico, en la inspiración del "óptimo de Pareto" que está a la base de la ponderación, tal que no se puede introducir ningún cambio más sin que se perjudique a alguien que ya había logrado el bienestar. La colisión de principios que reclama la ponderación, esto es la consideración de en qué grado y/o medida he de administrar el cumplimiento de un principio tal que a mayor cumplimiento de uno, menor cumplimiento del otro, expresa esa tensión que vemos en el justo medio aristotélico y que tiene como consecuencia y como supuesto a la vez, el respeto al principio de proporcionalidad. El justo medio para Aristóteles no es la mitad, ni el equilibrio que se logra en el medio exacto, no hay respuesta correcta que sea única, pero sí hay, al igual que en la ponderación el mayor grado posible de cumplimiento de una virtud y de un principio, en cada caso. Es una metodología que permite establecer la mejor acción posible y por ello conjuga el doble valor de la justicia como virtud y como administración.

- Racionalidad y relatividad: La virtud ha de ser el justo medio entre dos extremos y este ha de resultar de la deliberación racional que lleva a los hombres a considerar las acciones a realizar en el contexto puntual en el que estas tienen lugar. 
Es para Aristóteles condición de la racionalidad humana, pero fundamentalmente de lo humano: la excelencia del hombre es su alma intelectiva y por ello, actuar sin considerar su visión y análisis, es meramente reaccionar como lo harían los animales y por ello sería degradante. La acción ha de ser por lo tanto pensada y elegida voluntariamente, llevada a cabo con pleno conocimiento de lo que se está realizando, sólo así puede cumplir la función de ser moral. Lo mismo sucede en el caso de la decisión que resulta de la ponderación, ésta ha de poder ser justifiaca racionalmente a través de la argumentación jurídica que dé cuenta de las buenas razones que inspiraron la resolución del caso concreto. Tanto así que tampoco la resolución de cada caso será la solución grabada en piedra, sino que contará como antecedente para otros casos - cada caso será un universo en sí mismo- que reclamará la fundamentación de las decisiones tomadas a la luz de la resolución de la tensión entre principios. Sólo así la administración de justicia podrá revestirse de la seriedad que inspire seguridad jurídica, sometiéndose al control racional de sus intervenciones.

\section{Bibliografía}

Alexy, Robert "Sistema Jurídico, principios jurídicos y razón práctica", en Doxa 5, Universidad de Alicante, 1988, traducción de Manuel Atienza.

Alexy, Robert El concepto y la naturaleza del Derecho, Buenos Aires, Marcial Pons, 2008

Alexy, Robert Derecho y razón práctica, México, Editorial Fontamara, 1993

Amstrong, A.H., Introducción a la Filosofía Antigua, Buenos Aires, Eudeba, 1957

Aristóteles,Ética Nicomaquea, Madrid,Gredos, 2007

Aristóteles, Política, Madrid, Gredos, 2007

Atienza, Manuel, Introducción al Derecho, Barcelona, Barcanova, 1985

Atienza, Manuel, Bioética, Derecho y Argumentación, Bogotá, Palestra, 2004

Dworkin, Ronald Los derechos en serio, Barcelona, Editorial Ariel, 1984

Hart, H.L. El concepto del Derecho, Buenos Aires, AbeledoPerrot, 1963

Kelsen, HansTeoría Pura del Derecho, México, Porrúa, 1996

Platón, República, Buenos Aires, Eudeba, 1973 